\title{
High-pressure / High-temperature Synthesis and Characterization of Boron-doped Diamond
}

Natalia Dubrovinskaia ${ }^{\mathrm{a}, \mathrm{b}, \mathrm{c}}$, Leonid Dubrovinsky $^{\mathrm{b}}$, Nobuyoshi Miyajima $^{\mathrm{b}}$, Falko Langenhorst ${ }^{b}$, Wilson A. Crichton ${ }^{\mathrm{d}}$, and Hans F. Braun ${ }^{\mathrm{c}}$

${ }^{a}$ Lehrstuhl für Kristallographie, Physikalisches Institut, Universität Bayreuth, 95440 Bayreuth, Germany

${ }^{\mathrm{b}}$ Bayerisches Geoinstitut, Universität Bayreuth, 95440 Bayreuth, Germany

${ }^{\mathrm{c}}$ Experimentalphysik V, Physikalisches Institut, Universität Bayreuth, 95440 Bayreuth, Germany

${ }^{\mathrm{d}}$ European Synchrotron Radiation Facility, Grenoble 38043, France

Reprint requests to Dr. Natalia Dubrovinskaia. Fax: +49 (0)921/55-3769.

E-mail: natalia.dubrovinskaia@uni-bayreuth.de

Z. Naturforsch. 61b, 1561 - 1565 (2006); received September 3, 2006

Bulk samples (with volumes up to $\sim 7.5 \mathrm{~mm}^{3}$ ) of boron-doped diamonds (BDD) were synthesized by means of direct reaction between boron carbide and graphite in a multianvil apparatus at high pressures and high temperatures (HPHT). X-ray diffraction data revealed the presence in BDD of a very small amount of a highly boron-enriched phase $\left(\mathrm{B}_{50} \mathrm{C}_{2}\right)$ and traces of the $\mathrm{B}_{13} \mathrm{C}_{2}$ used as an initial material. The absence of $\mathrm{B}_{50} \mathrm{C}_{2}$ in the product of treatment of pure $\mathrm{B}_{13} \mathrm{C}_{2}$ under the same HPHT conditions suggests that boron-rich carbides exsolute from diamond on quenching leading to boron depletion of the diamond matrix. These observations imply that boron solubility in diamond increases at high pressure and high temperature. This result may have important implications for the understanding of the mechanism of boron incorporation into diamond at HPHT synthesis and for the interpretation of the data on superconductivity of polycrystalline BDD.

Key words: Boron-doped Diamond, High-pressure Synthesis, X-ray Diffraction

\section{Introduction}

Increasing interest in studies of doped diamonds is caused by the drastic influence of dopants on their physical properties, in particular, on the metalinsulator transition [1]. Artificially diamond can be doped using a few methods: doping during growth (by high-pressure / high-temperature (HPHT) methods or chemical vapor deposition (CVD) technique); doping by ion implantation or doping by thermal diffusion [2]. Ion implantation is problematic because of the thermal instability of diamond, in a process where high annealing temperatures (above $1700{ }^{\circ} \mathrm{C}$ ) are needed after implantation to get rid of the damage and to activate the implanted ions (i.e. to move those to substitutional sites). Diffusion processes are slow, so only very thin layers with high resistance have been produced using the thermal diffusion method [2]. Doping methods during crystal growth are most promising. The HPHT technique has an advantage due to the high speed of the process and a possibility to produce bulk homogeneous samples through the control of the components in the initial mixture.
Diamond doping with As, $\mathrm{P}$ and other atoms has been reported [3-5], but the small boron atom, which can be incorporated with high reproducibility and in high enough concentration to be useful for electronic devices, remains the only efficient dopant atom in bulk diamond [2]. Polycrystalline HPHT boron doped diamond attracts special attention due to the discovery of superconductivity [6] and the necessity to investigate and explain this physical phenomenon more deeply. However, little is known about the mechanism and the upper limit of doping achievable by this technique.

The purpose of the present study is to synthesize boron-doped polycrystalline diamonds using a multianvil apparatus at various $P T$ conditions and carefully investigate their phase composition and structure in order to shed light on the mechanism of boron incorporation into diamond.

\section{Experimental Section}

As starting materials in different experiments we used graphite (99.99\%, Goodfellow Inc.) and $\mathrm{B}_{13} \mathrm{C}_{2}(99.99 \%$, Goodfellow Inc.). The pure powders $\left(\mathrm{B}_{13} \mathrm{C}_{2}+\mathrm{C}\right.$-graphite) 


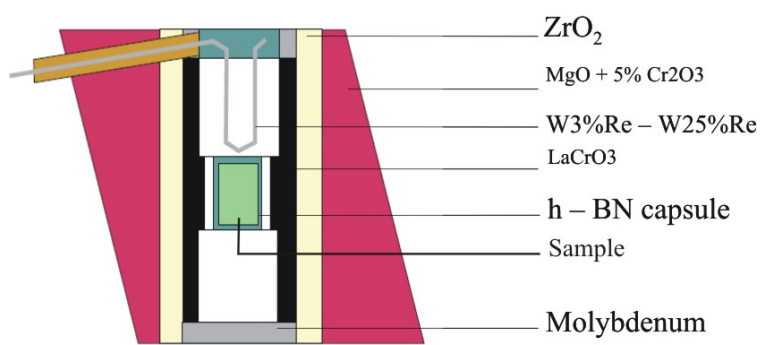

Fig. 1. The sample assembly for HPHT synthesis using a multianvil apparatus.

were mixed in a ratio $\mathrm{C}: \mathrm{B}=13: 1(\sim 7$ at. $\% \mathrm{~B})$ for the $\mathrm{BDD}$ synthesis. Pure $\mathrm{B}_{13} \mathrm{C}_{2}$ was also treated under the same $P T$ conditions as for the BDD synthesis.

HPHT experiments were conducted in a pressure range from 9 to $20 \mathrm{GPa}$ at temperatures $2500-2700 \mathrm{~K}$ using a twostage 6-8 type large-volume multianvil system with a Zwick 5000 tons press described elsewhere [7]. The sample assembly [8,9] (Fig. 1) consisted of a $\mathrm{MgO}\left(+5\right.$ wt. $\% \mathrm{Cr}_{2} \mathrm{O}_{3}$ ) octahedron with $18 \mathrm{~mm}$ edge length containing a $\mathrm{LaCrO}_{3}$ heater. The octahedron was compressed using $54 \mathrm{~mm}$ tungsten carbide anvils with a truncation edge length of $11 \mathrm{~mm}$ and pyrophyllite gaskets. The sample was placed into a $h$ BN capsule of a cylindrical shape with a sample chamber of $2 \mathrm{~mm}$ in diameter and $3 \mathrm{~mm}$ height. The sample temperature was monitored by a W3\%Re-W25\%Re thermocouple located axially with respect to the heater and with a junction close to the $h$-BN capsule, without corrections for the pressure effect on the thermocouple's emf. The sample pressure at high temperatures as a function of hydraulic oil pressure was calibrated using the $P / T$ diagrams [7] of $\mathrm{MgSiO}_{3}$ and $\mathrm{Mg}_{2} \mathrm{SiO}_{4}$. The pressure and temperature uncertainties were estimated to be $1 \mathrm{GPa}$ and $50 \mathrm{~K}$, respectively. Samples were gradually compressed to the desired pressure at ambient temperature, and then the temperature was stepwise increased (with a heating rate of about $100 \mathrm{~K} \mathrm{~min}^{-1}$ ) up to the desired value. Duration of heating was three to six minutes in different runs. The samples were quenched by switching off the power and then slowly decompressed. Samples came out as well-sintered cylinders of a black shining material in a $c$-BN capsule ( $h$-BN transformed to the cubic phase at high pressure and high temperature) of about $1.8 \mathrm{~mm}$ in diameter and $3 \mathrm{~mm}$ in height.

Quenched samples were studied by X-ray powder diffraction using a high-brilliance FRD RIGAKU diffractometer [10] $\left(\mathrm{Mo} K_{\alpha}\right.$ radiation, $60 \mathrm{~mA}, 55 \mathrm{kV}$, APEX CCD area detector) installed at the Bayerisches Geoinstitut and synchrotron radiation facilities at ESRF (ID30, with X-ray constant wavelength of $0.3738 \AA$ in angle-dispersive mode). Raman spectra were collected using the Raman spectrometers XY Dilor, operating with a $514 \mathrm{~nm}$ laser, and LabRam, operating with a $632 \mathrm{~nm}$ incident laser.
The chemical composition and texture of the samples were studied using a LEO-1530 scanning electron microscope (SEM) and a PHILIPS CM20 FEG analytical transmission electron microscope (ATEM), operating at $200 \mathrm{kV}$ equipped with a Gatan 666 PEELS system. For quantitative chemical microprobe analysis a Jeol (JXA-8200) WD/ED combined microanalizer with a beam of $2-3 \mu \mathrm{m}$ operating at an accelerating voltage of $20 \mathrm{keV}$ and a beam current of $50 \mathrm{nA}$ was used. Quantitative analysis for 3 elements was carried out using WDX. Analyzing crystals used for B, C, and $\mathrm{N}$ were LDEB, LDE2, and LDE1, respectively. Counting time was $10 \mathrm{~s}$. Reference standards were supplied by Jeol: boron nitride BN (99.999\% purity from Fulmer Research) and glassy carbon ( $>99.99 \%$ purity from Alfa).

\section{Results and Discussion}

The resolution of the energy-dispersive detector of the LEO-1530 scanning electron microscope is not sufficient for quantitative chemical analysis of B-C compounds. However, the presence of only two elements in all samples, carbon and boron, was confirmed. Quantitative microprobe analysis of the materials is difficult because of the rough surface of the hard samples [8,9]. Across small well-polished areas (about $300 \mu \mathrm{m}$ in diameter) we found homogeneous distribution of carbon and boron with the following average content of the elements: C 97.4(1.5) at.\%; B 2.6(0.6) at.\% for the sample synthesized at $20 \mathrm{GPa}$ and $2700 \mathrm{~K}$ (76 points, maximum deviation of the sum of boron and carbon from $100 \%$ is less than 2\%); C 98.2(1.8) at.\%; B 1.8(0.9) at.\% (69 points) for the sample synthesized at $9 \mathrm{GPa}$ and $2600 \mathrm{~K}$. The boron content in the sample synthesized at $20 \mathrm{GPa}$ is close to the value reported by Ekimov et al. [6] for samples synthesized at 8-9 GPa.

Our sample, synthesized at the same $P T$ conditions as those in [6], contains less boron. We would like to underline here that this is just a total amount of boron in the sample. The data provide no information about its location in diamond and/or impurities. This is a common problem of all methods applied to determine the boron content in BDD (secondary mass spectroscopy (SIMS) [11]; infrared transmission spectroscopy [12]; microprobe analysis [9]), which give the boron content in a limited volume of a sample (we would call it "bulk" boron content), and say nothing about the true boron location, in diamond (substitutional or interstitial) or in extra phases. Taking into account that conventional X-ray techniques do not allow determining the presence of impurities in very low 


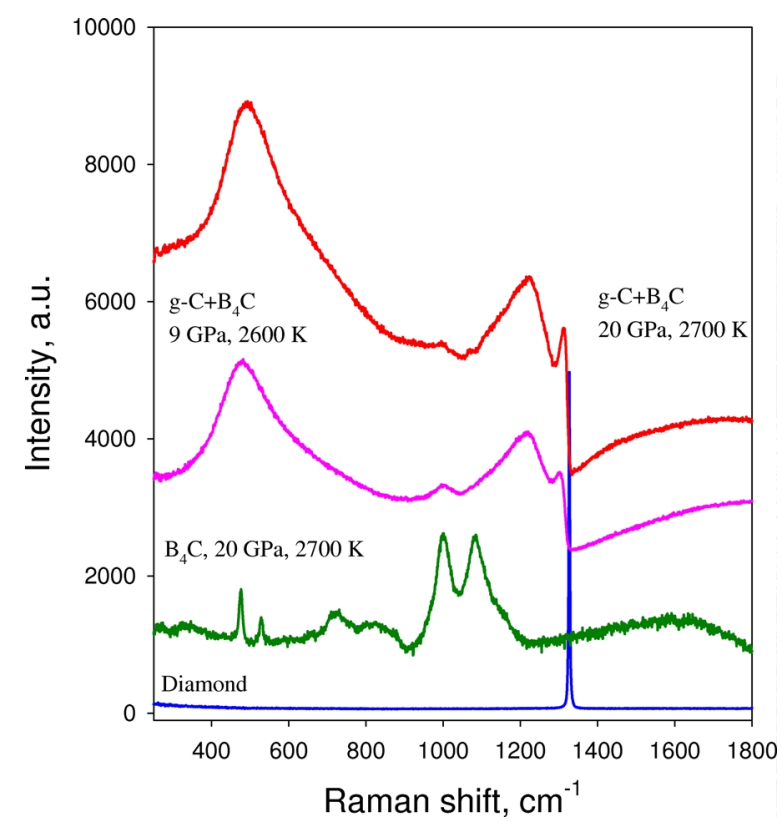

Fig. 2. Raman spectra of the boron doped diamond samples synthesised at different $P T$ conditions in comparison with the Raman spectrum of undoped diamond and $\mathrm{B}_{4} \mathrm{C}$ samples treated at $20 \mathrm{GPa}$ and $2700 \mathrm{~K}$.

concentrations, one cannot really judge which factors influence the unit cell parameters of BDD. This can explain why the attempts to quantify the "lattice parameter value - boron content" dependence $[11,12]$, which we have already discussed in [9], are inconsistent.

The present work provides a good illustration for this statement. Both studied samples have Raman spectra typical for BDD $[13,14]$ with concentrations of boron higher than $\sim 3 \times 10^{20} \mathrm{~cm}^{-3}(0.17$ at.\%) (Fig. 2). The Raman spectra of the samples synthesized at $9 \mathrm{GPa}$ and $20 \mathrm{GPa}$ are dominated by the phonon modes at $495,998,1220,1303 \mathrm{~cm}^{-1}$ and 496, 998, 1223, $1312 \mathrm{~cm}^{-1}$, respectively. In [15] "a sample-average Debye temperature $\Theta_{D}=1440 \mathrm{~K}$, which is about $23 \%$ smaller than the Debye temperature of undoped diamond" (cited from [15]) was calculated for a BDD sample using experimental data on specific heat measurements. The authors [15] claimed that this $23 \%$ difference in the Debye temperature indicates lattice vibrations softening in response to doped holes. If so, the Raman line of BDD should move towards lower values with respect to the position of the characteristic diamond Raman line $\left(1332 \mathrm{~cm}^{-1}\right)$ with increasing boron concentration. However, the line at $\sim 1303 \mathrm{~cm}^{-1}$ of the lower pressure sample ( $9 \mathrm{GPa}$, with lower boron con- (a)

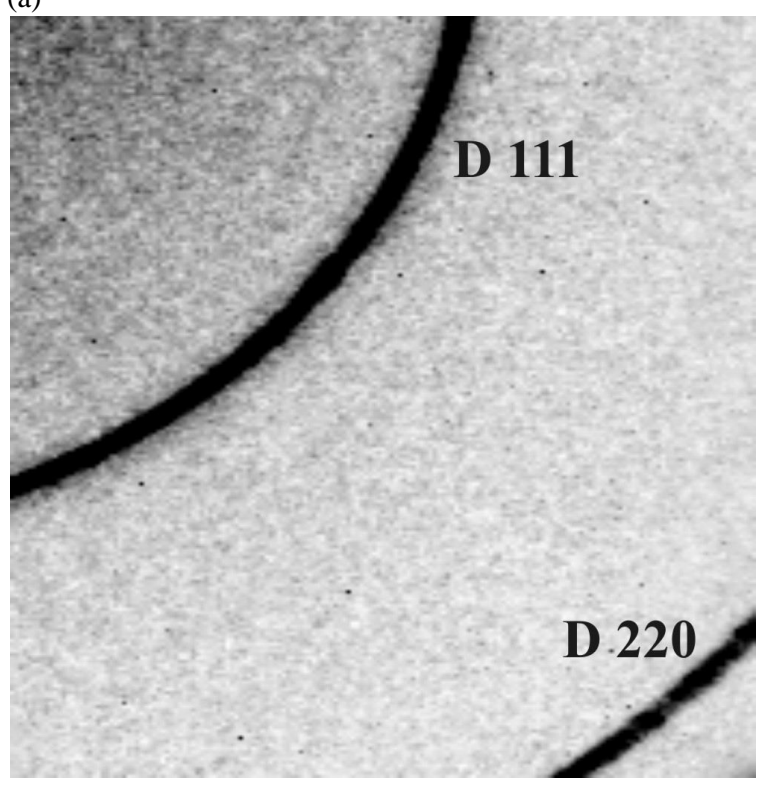

(b)

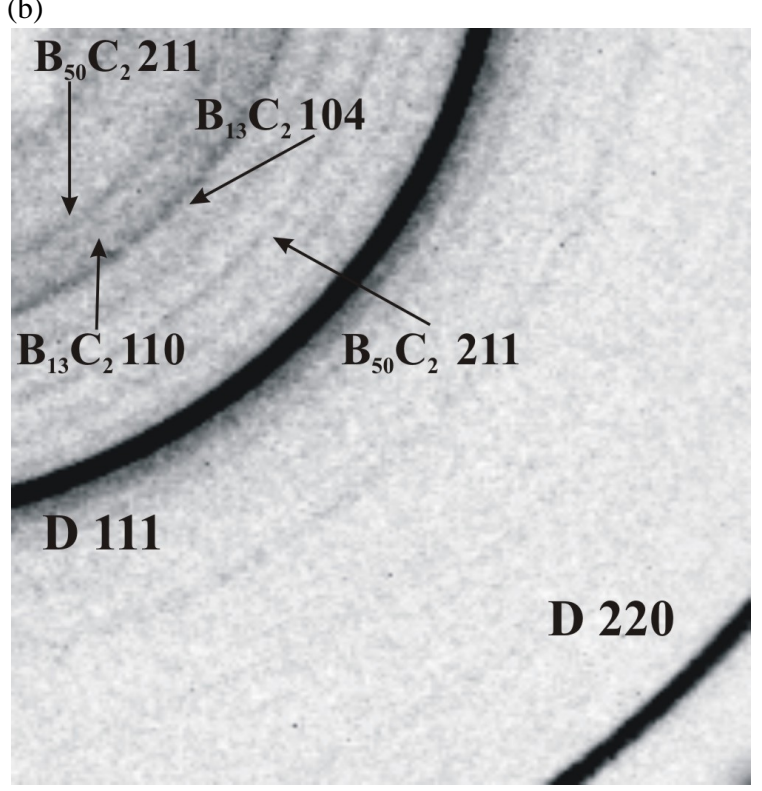

Fig. 3. Parts of the 2D diffraction images of the sample recovered after an experiment at $20 \mathrm{GPa}$ and $2700 \mathrm{~K}$ obtained after (a) $30 \mathrm{~s}$ and (b) $1800 \mathrm{~s}$ exposure. D denote diamond diffraction lines; $\mathrm{B}_{13} \mathrm{C}_{2}$ and $\mathrm{B}_{50} \mathrm{C}_{2}$ denote diffraction lines of extra boron-rich phases.

tent) moved to the opposite direction $\left(\sim 1312 \mathrm{~cm}^{-1}\right)$ expected from the apparent higher boron concentration (measured on microprobe) for the $20 \mathrm{GPa}$ sample. A maximal $2.5 \%$ shift of the BDD line with respect to the $1332 \mathrm{~cm}^{-1}$ undoped diamond line also does not corre- 

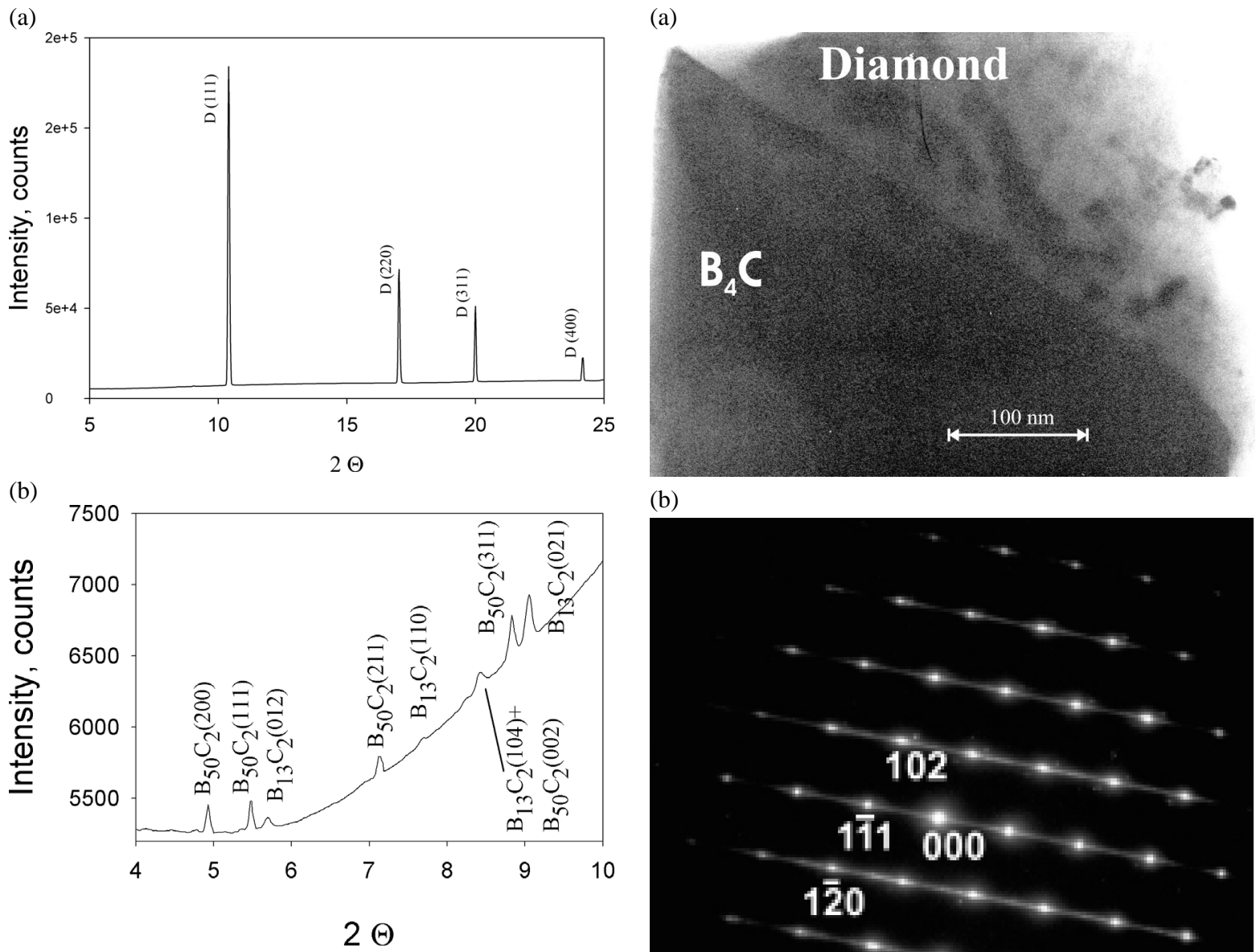

(b)

Fig. 4. (a) Synchrotron X-ray diffraction pattern of the sample synthesized at $20 \mathrm{GPa}$. (b) The enlarged area between 4 and 10 degrees $2 \Theta$ with reflections of boron-rich phases $\left(\mathrm{B}_{50} \mathrm{C}_{2}\right.$ and $\left.\mathrm{B}_{13} \mathrm{C}_{2}\right)$ clearly visible.

late with the $23 \%$ decrease of the Debye temperature reported in [15].

The lattice parameters $3.57740(16) \AA$ and $3.57250(31) \AA$ for $9 \mathrm{GPa}$ and $20 \mathrm{GPa}$ samples, respectively, are larger than that of pure diamond (3.5667 A, JCPDS No. 6-0675) in agreement with literature data $[11,12,16]$ for $\mathrm{BDD}$, but they also do not correlate with the apparent boron content in the samples.

Fig. 3 shows the diffraction patterns of the sample recovered after the experiment at $20 \mathrm{GPa}$ and $2700 \mathrm{~K}$. Only diamond lines are present in Fig. 3a, which shows a diffraction pattern obtained after $30 \mathrm{~s}$ exposure. However, the increase of the exposure time to $1800 \mathrm{~s}$ revealed the presence of extra reflections (Fig. 3b). Synchrotron X-ray data confirmed the presence of impu-

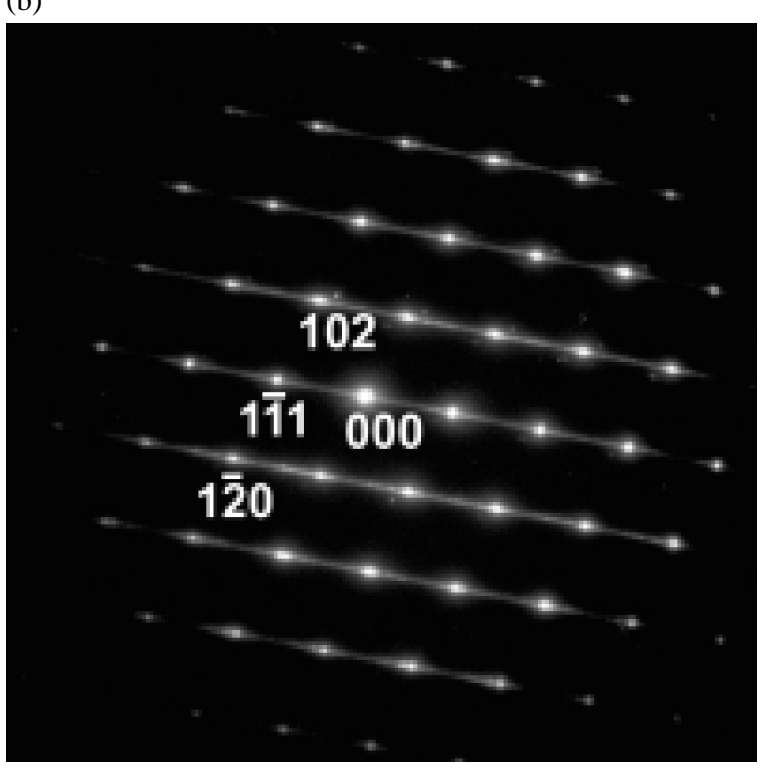

Fig. 5. (a) A bright field TEM image of a region containing boron doped diamond and $\mathrm{B}_{4} \mathrm{C}$. (b) A selected area electron diffraction pattern of $\mathrm{B}_{4} \mathrm{C}$ with (101) hex-twinning and staking faults, which confirms the presence of this carbide in the BDD samples.

rities (Fig. 4) and allowed us to identify them as the boron-rich carbides $\mathrm{B}_{50} \mathrm{C}_{2}$ and $\mathrm{B}_{13} \mathrm{C}_{2}$ (the latter was used as a source of boron in the BDD synthesis). $\mathrm{B}_{50} \mathrm{C}_{2}$ was found long ago [17] and pyrolytically synthesized in a reproducible manner [18,19] (ICSD \#9734), but it has not been reported so far as a product of HP synthesis. Boron carbide $\left(\mathrm{B}_{13} \mathrm{C}_{2}\right)$ is a nonstoichiometric compound. Its carbon content can vary from 9.88 to 23.4 at.\% [20]. EELS analysis gave us the boron carbide's chemical composition corresponding to the for- 
mula $\mathrm{B}_{5.26} \mathrm{C}$. The whole series of nonstoichiometric boron carbides is usually denoted by the formula $\mathrm{B}_{4} \mathrm{C}$ used for simplicity in the figures.

In the samples synthesised at lower pressure, the same impurities were found with the only difference that reflections of $\mathrm{B}_{13} \mathrm{C}_{2}$ were clearly observed in the $\mathrm{X}$-ray diffraction patterns even after a very short exposure and despite the lower total boron content shown by microprobe data for this sample. TEM images and selected area electron diffraction patterns (Fig. 5) confirmed the presence of $\mathrm{B}_{13} \mathrm{C}_{2}$ in the samples.

These observations raise the question about the mechanism of the formation of boron-rich phases as impurities in BDD. To clarify this point we subjected pure $\mathrm{B}_{13} \mathrm{C}_{2}$ to the HPHT treatment under the same conditions as those applied for BDD synthesis. We did not observe any changes or any presence of extra phases in $\mathrm{B}_{13} \mathrm{C}_{2}$ [10] after HPHT treatment. This means that
$\mathrm{B}_{50} \mathrm{C}_{2}$ does not form directly from $\mathrm{B}_{13} \mathrm{C}_{2}$. Boron from $\mathrm{B}_{13} \mathrm{C}_{2}$ is likely to be first incorporated into diamond during transformation of carbon to diamond at high pressures and temperatures. Thus, the possible mechanism of formation of boron-rich phases may be their exsolution from the BDD matrix on quenching with simultaneous depletion of the matrix of boron. However, the reason for spontaneous boron segregation still remains unclear.

In this context it seems noteworthy that knowledge of the true phase composition of polycrystalline samples of BDD is very important both for a target synthesis of BDD with a certain level of doping, and for a correct interpretation of the properties of the products including the superconductivity phenomenon.

\section{Acknowledgement}

Financial support by the Deutsche Forschungsgemeinschaft (DFG) is gratefully acknowledged.
[1] T. Tshepe, C. Kasl, J. F. Prins, M. J. R. Hoch, Phys. Rev. B 70, 245107 (2004).

[2] K. Thonke, Semicond. Sci. Technol. 18, S20 (2003).

[3] T. P. Chow, R. Tyagi, IEEE Trans Electron. Dev. 41, 1481 (1994).

[4] S. Koizumi, T. Teraji, H. Kanda, Diam. Relat. Mater. 9, 935 (2000).

[5] Z. Teukam, J. Chevallier, C. Saguy, R. Kalish, D. Ballutaud, M. Barbe, F. Jomard, A. Tromson-Carli, C. Cytermann, J.E. Butler, M. Bernard, C. Baron, A. Deneuville, Nature Materials 2, 482 (2003).

[6] E. A. Ekimov, V.A. Sidorov, E. D. Bauer, N.N. Mel'nik, N. J. Curro, J. D. Thompson, S. M. Stishov, Nature 428, 542 (2004).

[7] D. J. Frost, B.T. Poe, R. G. Tronnes, C. Liebske, A. Duba, D. C. Rubie, Physics of the Earth and Planetary Interiors 143-144, 507 (2004).

[8] V. Solozhenko, N. A. Dubrovinskaia, L. S. Dubrovinsky, Appl. Phys. Lett. 85, 1508 (2004).

[9] N. Dubrovinskaia, G. Eska, G. A. Sheshin, H. F. Braun, J. Appl. Phys. 99, 033903-1-7 (2006).

[10] L. Dubrovinsky, N. Dubrovinskaia, I. Kantor, F. Nestola, D. Gatta, High Pressure Research 26, 2, 137 (2006).
[11] E. Bustarret, E. Ghaeeraert, K. Watanabe, Phys. Stat. Sol. (a) 199, 9 (2003).

[12] F. Brunet, P. Germi, M. Pernet, A. Deneuville, E. Ghaeeraert, F. Laugier, M. Burdin, G. Rolland, Diamond and Related Materials 7, 869 (1998).

[13] F. Pruvost, E. Bustarret, A. Deneuville, Diamond and Related Materials 9, 295 (2000).

[14] R. J. Zhang, S. T. Lee, Y. W. Lam, Diamond and Related Materials 5, 1288 (1996).

[15] V.A. Sidorov, E. A. Ekimov, S. M. Stishov, E. D. Bauer, J.D. Thompson, Phys. Rev. B 71, 060502 (2005).

[16] O. A. Voronov, A. V. Rakhmanina, Inorg. Mater. 29, 533 (1993).

[17] J. L. Hoard, R. E. Hughes, D. E. Sands, J. Amer. Chem. Soc. 80, 4507 (1958).

[18] K. Ploog, H. Schmidt, E. Amberger, G. Will, K. H. Kossobutzki. J. Less-Common Met. 29, 161 (1972).

[19] G. Will, K. Ploog, Nature 251, 406 (1974).

[20] P. S. Kisliy, M. A. Kuzenkova, N. I. Bondaruk, B. L. Grabchuk, Boron carbide. Naukova Dumka, Kiev (1988), 216 p. (in Russian). 